Bull. Mater. Sci., Vol. 8, No. 2, May 1986, pp. 155-167. (C) Printed in India.

\title{
Computer simulation of defects and radiation damage
}

\author{
K KRISHAN, B PURNIAH and S SRINIVASAN \\ Materials Science Laboratory, Indira Gandhi Centre for Atomic Research, Kalpakkam \\ 603102 , India
}

\begin{abstract}
In this paper, we describe the computer simulation technique and its application to the study of radiation damage. Details of two important methods: the static and the dynamic methods have been discussed. Applications to the study of point defect formation and stability, their clusters, diffusion, dislocations and dislocation-point defect interaction are discussed drawing from our own work wherever possible. A short mention is made of the importance of the interatomic potential. Examples for the case of magnesium and other hcp metals, bcc iron and $\mathrm{fcc} \mathrm{Ni}$ are cited and numbers for various quantities like formation energy, dipole tensor, interaction energy etc are quoted.
\end{abstract}

Keywords. Computer simulation; interatomic potential; static method; dynamic method; point defects; dislocations; displacement damage.

\section{Introduction}

The study of matter in radiation environments has developed into an important area of research due to the technological demand of finding reliable and stable materials for the structural components of nuclear reactor systems. The energetic particles of the radiation displace the atoms in a metal (or alloy) bringing about irreversible changes in the composition and microstructure. These changes at an elementary level involve three processes: the formation of defects, their diffusion and subsequently annihilation. Each of these damage events leaves behind a permanent mark whose cumulative effect results in the changes in alloy properties often referred to as radiation damage. Some examples of these are embrittlement, irradiation creep and swelling. Invariably, these involve the redistribution of radiation-produced defects due to diffusion and are studied with the help of rate equations whose parameters are related to basic 'damage events'. It is in the study of this more basic aspect of radiation damage that computer 'experiments' play a very important role.

The basic damage process occurs over several orders of magnitude in time. In the first $10^{-17} \mathrm{sec}$ an energetic projectile transfers its energy to the nucleus of the metal lattice atom producing a primary knock-on-atom (PKA). In the next $10^{-13} \mathrm{sec}$ the PKA slows down creating a cascade of secondary displacements. The cascade volume dissipates its energy in $10^{-11} \mathrm{sec}$ forming unstable defects which spontaneously rearrange themselves into stable defects. Then begins a long period of several hundreds of seconds when these defects diffuse and eventually annihilate at sinks. This annihilation, or defect annealing, depends on the interaction between the defects and leads to the formation of clusters or complexes depending on their relative stability. The very short times involved and the statistical nature of the events (due to the large number of degrees of freedom) makes it difficult to use analytic methods of investigation or device experiments where each event can be followed. However, the advent of high speed 
computers has made it possible to simulate these processes in a way as if they are actually occurring in a solid. It is for this reason that such studies have been termed computer experiments.

Computer experiments deal either with the dynamic aspects of damage like the formation of cascades or with static aspects which concern the formation of stable defect configurations. In $\S 2$, we give an account of the computer simulation procedure for both static and dynamic experiments. Section 3 deals with the range of problems which have been studied with typical examples and results drawn from our own work wherever possible. Finally in $\$ 4$ we give a brief conclusion.

\section{Computer simulation technique}

In general, in all computer simulation methods, a model crystallite of the required material is constructed in which the atoms are allowed to interact via a suitable interatomic potential. The defect is then placed at the centre of this crystallite. The presence of the defect will cause forces to act on the atoms surrounding it. This configuration is allowed to relax using either the static or dynamic method. The number of atoms in this crystallite usually ranges from a few hundred to a few thousand depending on the complexity of the defect under study and the range of interatomic potential. The computation of the various quantities of interest requires that a neighbour set be generated for each atom in the crystallite. While the generation of the crystallite itself is quite simple once the crystal type is known, generation of neighbours calls for greater efforts and will be treated in greater detail below. After a brief discussion on interatomic potentials we discuss the techniques for relaxation. Several techniques used in computer simulation including sample programmes are discussed in a recent book by Beeler (1983a). Towards the end of this section two specific examples viz the simulation of point defects and dislocations is discussed in slightly greater detail.

\subsection{Generation of neighbours}

In the case of cubic crystals the position vector of any atom can be conveniently described by a set of three integers if distances are measured in half lattice units. The neighbour positions in an fcc crystal can be generated by a technique of partitioning the squares of the radial distances $N R^{2}$ (which occur in multiples of 2) and thereafter by taking the various possible permutations of these partitioned numbers. Thus we have $N R^{2}=N X^{2}+N Y^{2}+N Z^{2}$ where the integers $N X, N Y$ and $N Z$ represent the components along the $x, y$ and $z$ directions respectively (see table 1). A given value of $N R^{2}$ denotes a particular shell and the atoms in the shell can be obtained by the permutations of the three numbers as shown in the table.

The generation of neighbours in an hcp lattice is more complicated. Firstly, the radius vector of all the atoms in the crystallite is calculated from a given origin and arranged in an ascending order. Atoms having the same radius are grouped together into one shell. These atom co-ordinates are then normalized to yield integer numbers and these are stored for later use. It may be noted that a further complication arises because of the c/a ratio. For instance the fourth and sixth neighbour shells of $\mathbf{M g}$ (c/a $=1.63)$ and $\mathrm{Zr}(\mathrm{c} / \mathrm{a}=1.86)$ get interchanged. 
Table 1. Shell-wise neighbour generation for an fcc crystal

\begin{tabular}{lcccrrr}
\hline$N R^{2}$ & $N X$ & $N Y$ & $N Z$ & $X$ & $Y$ & $Z$ \\
\hline 0 & 0 & 0 & 0 & 0 & 0 & 0 \\
2 & 0 & 1 & 1 & 0 & 1 & -1 \\
& & & & 0 & -1 & 1 \\
4 & 0 & 0 & 2 & 0 & -1 & -1 \\
& & & & 1 & 0 & 1 \\
6 & 1 & 1 & 2 & 1 & 0 & -1 \\
& & & & -1 & 0 & 1 \\
8 & 0 & 2 & 2 & -1 & 0 & -1 \\
& & & & 1 & 1 & 0 \\
10 & 0 & 1 & 3 & 1 & -1 & 0 \\
& & & & -1 & 1 & 0 \\
12 & 2 & 2 & 2 & -1 & -1 & 0 \\
& & & & $:$ & $:$ & $:$ \\
\hline
\end{tabular}

While the above discussion holds for the simulation of point defects, in the case of dislocation simulation, it is convenient to choose the co-ordinate axes along the dislocation line and Burgers vector thereby necessitating a transformation in neighbour co-ordinates.

\subsection{Interatomic potential}

A crucial aspect of computer simulation is the choice of an appropriate interatomic potential. A large number of diverse interatomic potentials have been proposed in the literature. These can be broadly classified as empirical and pseudopotentials. Examples of the former are the Morse, Lennard-Jones and several polynomial potentials (Johnson 1973). Pseudopotentials are known to represent the interaction better but these have not made much headway in defect calculations. The most widely used polynomial potentials for defect calculations have been the cubic and the quintic splines where the polynomial coefficients are obtained by least squares spline fitting the potential to the elastic constants of the material. Such potentials for cubic metals have been described by Baskes and Melius (1979) while those for hcp metals have been described by Purniah et al (1980).

\subsection{Relaxation techniques}

As already stated, the configuration of the crystallite containing the defect is allowed to relax to a minimum energy configuration. The crystallite is usually divided into two regions to save on computation time. In the inner region I, the atoms are allowed to relax under the influence of the chosen potential. The atoms in the outer region II are constrained to their perfect lattice positions as shown in figure 1 . The relaxation of atoms in region I can be done either by the static method or the dynamic method. In the static method, the relaxation is performed by an iterative technique using the Newton- 


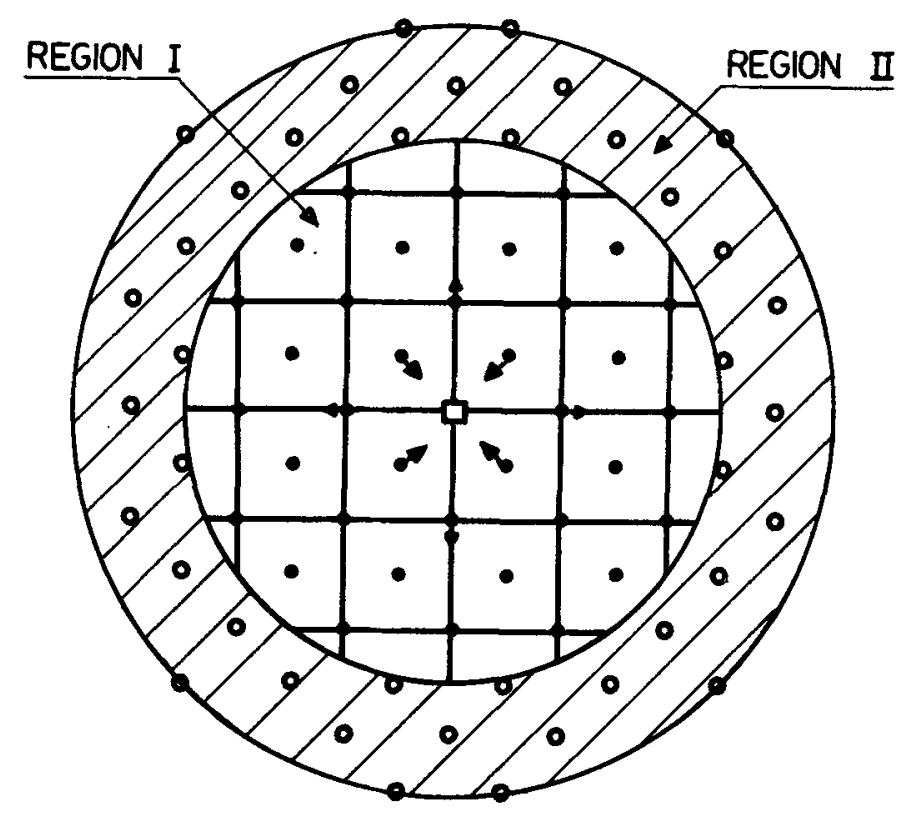

Figure 1. Schematic representation of the two regions in a crystallite used in computer simulation studies. Open circles represent atoms which are fixed while filled circles indicate the atoms which are allowed to move. Also shown by arrows is the displacement of atoms around a vacancy ( $\square$ ) placed at the centre.

Raphson method. Thus the relaxed position of the $n$th atom after $k+1$ iterations is given by

$$
X_{k+1}(n)=X_{k}(n)-\propto \frac{\partial U}{\partial X(n)}\left|\frac{\partial^{2} U}{\partial X^{2}(n)}\right|_{X(n)=X_{t}(n)},
$$

where $X_{k}(n)$ is the co-ordinate of the $n$th atom after the $k$ th iteration and $U$ is the configuration energy. The convergence factor $\alpha(0<\alpha \leqslant 1)$ has been introduced to damp out the oscillations which are usually present in the co-ordinates $X_{k}$ while applying the Newton-Raphson technique directly. The total number of iterations for reasonable convergence may run into several hundred depending on the problem being considered. From the above discussion it is clear that the simulation work requires a large and fast computer. Typically all the programs operate in double precision, occupying $\mathbf{S 1 2}$ kilobytes of core memory and take upto one hour of CPU time.

In the dynamical method also referred to as "molecular dynamics" method, the movement of individual atoms in an assembly is followed using Newton's equations. Given the initial position and the velocity for each atom in the crystallite, the dynamical history is followed by numerically integrating the $3 N$ simultaneous equations of motion for the co-ordinate components of the $N$ atoms in the crystallite. In the process, the $3 N$ velocity components for the $N$ atoms are also determined.

The dynamical method is very useful in describing temperature effects on Frenkel pair production, annihilation and point defect complexes.

The net force $F(t)$ experienced by an atom at time $t$ is determined by the relative positions of all the other atoms at time $t$ and $a(t)=F(t) / m$. Given $a(t)$, the advanced 
time quantities are given by using one of the many schemes available (Gibson et al 1960)

$$
\begin{aligned}
v(t+\Delta t / 2) & =v(t-\Delta t / 2)+a(t) \Delta t \\
x(t+\Delta t) & =x(t)+v(t+\Delta t / 2) \Delta t .
\end{aligned}
$$

In these simulations, the value of $\Delta t$ is in the region of $10^{-14} \mathrm{sec}$ which gives an idea of the time scales which can be probed.

\subsection{Simulation of point defects and dislocations}

In the case of point defect simulation, the inner region $I$ is chosen to be a sphere. Periodic boundary conditions are employed to essentially generate the large size of the assembly needed to remove any surface effects. This is further checked out by varying the size of region I to see the effect of the boundary region. The defect formation energy is calculated by subtracting the energy of the perfect lattice from the energy of the relaxed lattice with either an extra atom (vacancy case) or an atom-less (interstitial case) at the crystal surface. The relaxation energy is obtained by subtracting the energy of the crystallite with the defect in the relaxed condition from that in the unrelaxed condition.

The inner region for the case of dislocation simulation is chosen to be cylindrical to take advantage of the symmetry in the problem. The direction of the dislocation coincides with the cylinder axis and periodicity along the dislocation line is assumed. The 'introduction' of the dislocation is done by imparting elastic displacements to the atoms by considering a dislocation at the centre of the block (Cotterill and Doyama 1967). The core configuration and other parameters can be obtained by the relaxation technique described. There have been several modifications to the methods described above for dislocations. One of these is the 'flexible' boundary condition method (Hoagland et al 1976) where the atoms in region II are moved in response to the changes which occur in the inner region. This enables a smaller crystallite size to be chosen thereby reducing the computation time. It may however be noted that in the study of the dislocation point defect interaction the flexible boundary methods cannot be used since the introduction of the point defect destroys the symmetry in the problem.

\section{Application of computer simulation to radiation damage problems}

In this section we briefly discuss some of the areas where computer simulation has been successfully used to investigate the behaviour and properties of defects produced by radiation. The selection has been made to cover a range of problems and to emphasize the nature of information obtained by the technique rather than the detailed results.

\subsection{Vacancies, interstitials and their clusters and complexes}

The study of point defects and their clusters has been one of the important areas where computer simulation experiments have been particularly successful. Here due to the many degrees of freedom available one has to examine from amongst several possibilities, the configurations which would be most stable. For example figure 2 shows three configurations each of di-vacancies and tri-vacancies and the three possible sites for a self-interstitial-namely split, tetrahedral and octahedral for an hcp lattice. 


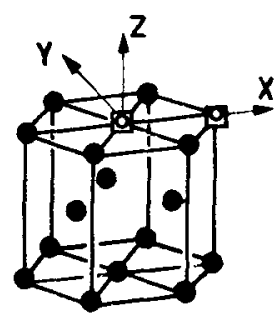

(a)

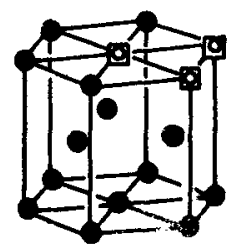

(d)

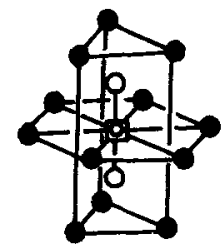

(g)

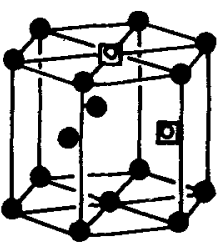

(b)

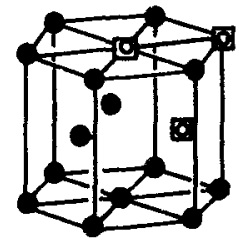

(e)

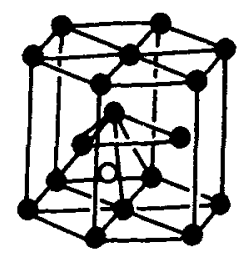

(h)

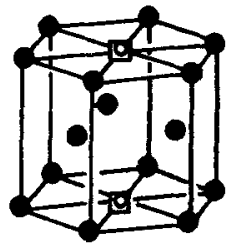

(c)

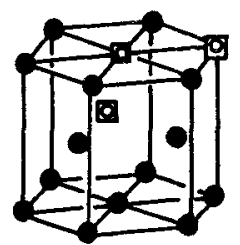

(

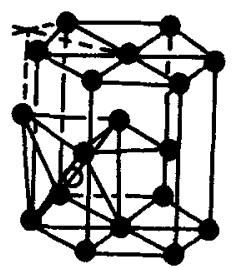

(i)

Figure 2. The three possible configurations each of the divacancy $(\mathbf{a}, \mathbf{b}, \mathbf{c})$ and trivacancy (d, e, f) in hep magnesium. The three configurations at the bottom are those for the split, tetrahedral and octahedral interstitial.

These are amongst the more favourable ones and further possibilities cannot be ruled out. Computer simulation has shown that for self-interstitials the split configurations along $\langle 110\rangle$ for bcc, $\langle 100\rangle$ for foc and $\langle 0001\rangle$ for hcp are most favourable on the basis of minimum energy consideration. A comparison of the strain field of a vacancy and a self-interstitial is shown in figure 3 for hcp Mg as a typical example (Sahu et al 1980). Table 2 shows the relaxation field around a monovacancy for several hcp metals (Purniah et al 1980). Characteristic of this strain field are the positive and negative displacements of the different neighbouring shells; the maximum displacement at the third neighbour for a vacancy in $\mathbf{M g}$; nearly an order of magnitude higher strain field for self-interstitials as compared to vacancies. Similar features have been seen in bcc iron and foc nickel which are some of the metals extensively studied. Very similar to these are investigations on over-sized and under-sized substitutional impurities whose strain fields show similar behaviour. For a $10 \%$ over-sized impurity in boc iron, the energy of each of the first-neighbour atoms increases $(+0.036 \mathrm{eV})$ (i.e. high energy atom site) and that of the second neighbours decreases $(-0.026 \mathrm{eV})$ (low energy atom site). A systematic study of this feature leads to a rule for predicting the geometry of an impurity-vacancy complex (Beeler 1983b). If a high-energy atom site in the substitutional impurity atom displacement field is replaced by a vacancy, the resulting 

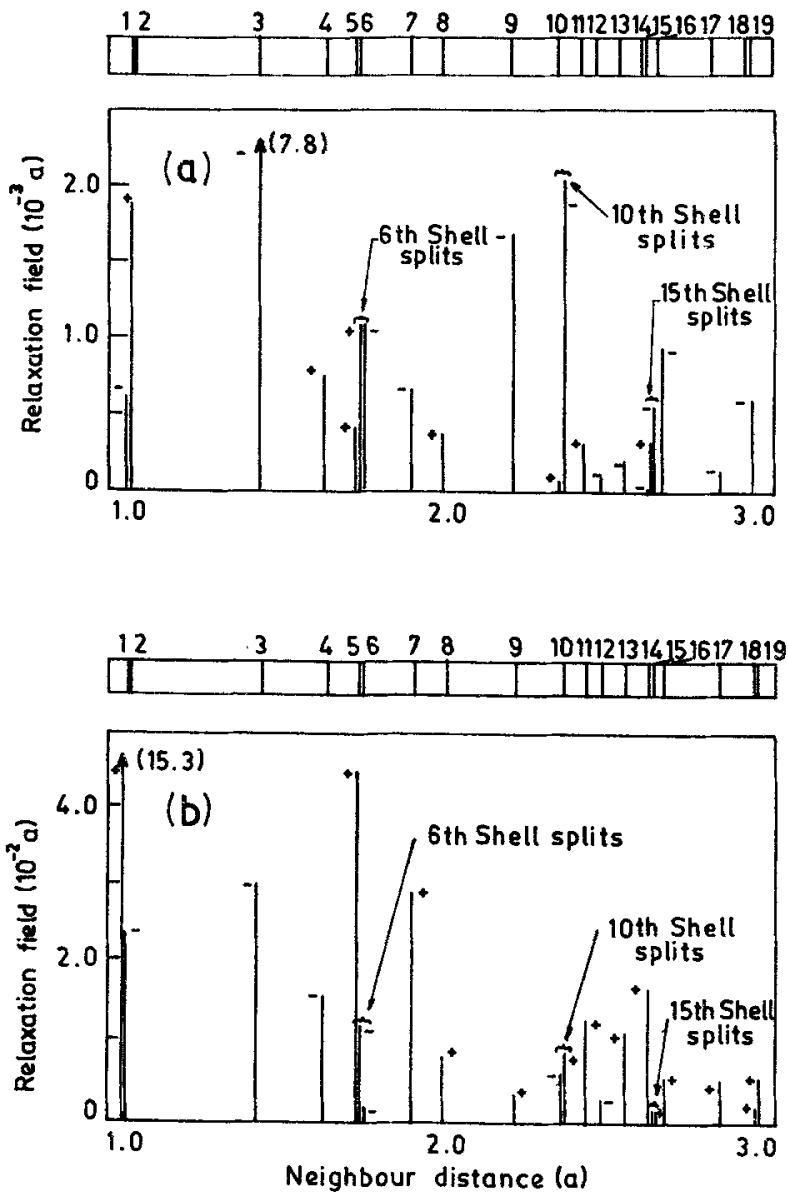

Figure 3. The relaxation field of all shells upto nineteen neighbours around a single vacancy (a) and a split interstitial (b). The bars on top represent the neighbour positions shown separately for clarity. (+ and - signs indicate outward and inward relaxation).

Table 2. Relaxation field around a monovacancy. The numbers 1 st, $2 \mathrm{nd}$, 3rd and 4 th refer to neighbours. (The + and - signs indicate outward and inward relaxation).

\begin{tabular}{|c|c|c|c|c|c|c|c|}
\hline \multirow[b]{2}{*}{ Metal } & \multicolumn{4}{|c|}{ Relaxation field $\left(10^{-2} \mathrm{a}\right)$} & \multirow{2}{*}{$\begin{array}{c}\text { Relaxation } \\
\text { volume } \\
\Delta V / V_{\text {at }}\end{array}$} & \multicolumn{2}{|c|}{ Dipole tensor $(\mathrm{eV})$} \\
\hline & ist & $2 n d$ & 3rd & 4 th & & $P_{11}=P_{22}$ & $P_{33}$ \\
\hline Hf & -1.32 & -0.65 & +0.48 & +0.41 & $-0 \cdot 18$ & $-4 \cdot 09$ & -7.94 \\
\hline $\mathbf{M g}$ & -0.40 & -0.34 & -0.69 & +0.01 & -0.09 & -0.93 & $-0 \cdot 95$ \\
\hline $\operatorname{Re}$ & -0.80 & -0.70 & +0.12 & $+0 \cdot 19$ & -0.14 & $-8 \cdot 16$ & $-11 \cdot 24$ \\
\hline $\mathrm{Y}$ & -2.09 & +0.70 & $+1 \cdot 36$ & +0.48 & -0.21 & -1.57 & -6.32 \\
\hline$\alpha-\mathbf{Z r}$ & -1.62 & -1.80 & +0.28 & +0.35 & -0.31 & -6.92 & $-8 \cdot 36$ \\
\hline $\mathrm{Be}$ & $+6 \cdot 15$ & +2.04 & +5.65 & $+3 \cdot 72$ & +0.56 & $+7 \cdot 74$ & +1.73 \\
\hline
\end{tabular}


complex will have a positive binding energy while for a low-energy atom it will result in a negative binding energy. Such features are also found in the stability of some defect complexes. For example in bcc iron a second neighbour arrangement of vacancies has been found to be more stable than first neighbours arrangement. In hcp Mg, of the three configurations (a), (b) and (c) shown in figure 2 for di-vacancies the formation (and binding) energies are 1.377 (0.086), 1.379 (0.084), 1.386 (0.077) eV'respectively showing that configurations (a) and (b) are likely to be more stable at lower temperatures (Sahu et al 1980). Similarly for the tri-vacancy configurations (d), (e) and (f) have energies $1.939(0.256), 1.942(0.252)$ and $1.972(0.222) \mathrm{eV}$ which favours the planar configuration (d). Extensions of such simulation experiments have been carried out to very large vacancy clusters $(\sim 40)$ to examine how such clusters could develop into vacancy dislocation loops or voids (Beeler and Johnson 1967).

Apart from substitutional impurities, light atoms like $\mathrm{C}, \mathrm{N}$ and $\mathrm{O}$ often occupy interstitial locations. The simulation technique in this case is very similar to that for selfinterstitials, the important difference arises from the use of an impurity-host potential. Apart from the defect formation energy $E_{f}$ and migration energy $E_{m}$, other important parameters are the defect formation volume and the dipole tensor. Results for some of the calculations are shown in table 3 (Purniah et al 1982). The agreement between the estimated $E_{m}$ and that obtained from experiments is seen to be very good. Further the dipole tensor values compare very favourably in sign and magnitude from those obtained by anelastic data and $x$-ray measurements.

Studying the behaviour of $\mathrm{He}$ in metals has been a problem of great technological interest where computer experiments have been very valuable. Helium can diffuse interstitially and readily forms complexes with vacancies. Computer simulation studies have revealed that as many as six to seven helium atoms can be accommodated in a single vacancy! These studies give detailed information about helium and vacancy binding to a complex of $n$ helium atoms and $m$ vacancies (Wilson et al 1976). The structure of some typical complexes is given in figure 4.

The study of diffusion of vacancies, interstitials, clusters and complexes of defects is an area where computer simulation is particularly useful. A defect moving from one equilibrium position to another has to pass through an intermediate unstable saddle point which determines the height for the migration barrier. Computer simulation provides detailed information about these intermediate configurations. As an example figure 5 shows the results of computer simulation of vacancy migration in bcc iron. The nature of the interaction and the positions of the surrounding atoms gives rise to two saddle points SP1 and SP2 as the atom A moves from its site to the vacancy (Beeler 1983c).

Table 3. Simulation results for various quantities for interstitials in $\alpha$-Fe, $\mathrm{Zr}$ and $\mathrm{Hf}$

\begin{tabular}{lccccrr}
\hline Interstitials & $\begin{array}{c}E_{i}^{f} \\
(\mathrm{eV})\end{array}$ & $\begin{array}{c}V_{f} \\
\left(\Omega_{0}\right)\end{array}$ & $\begin{array}{c}E_{m} \\
(\mathrm{eV})\end{array}$ & $\begin{array}{c}E_{m}(\mathrm{expt}) \\
(\mathrm{eV})\end{array}$ & \multicolumn{2}{c}{ Dipole tensor $(\mathrm{eV})$} \\
\hline $\mathrm{C}$ in $\alpha-\mathrm{Fe}$ & 1.35 & 0.055 & 0.86 & 0.835 & -0.053 & 4.223 \\
$\mathrm{C}$ in $\mathrm{Zr}$ & 1.38 & 0.048 & 1.48 & 1.48 & 1.472 & 1.395 \\
$\mathrm{O}$ in $\mathrm{Zr}$ & 2.98 & -0.002 & 1.94 & 2.04 & -0.049 & -0.046 \\
$\mathrm{O}$ in $\mathrm{Hf}$ & 3.30 & 0.0 & 2.16 & 2.26 & 0.001 & 0.001 \\
\hline
\end{tabular}



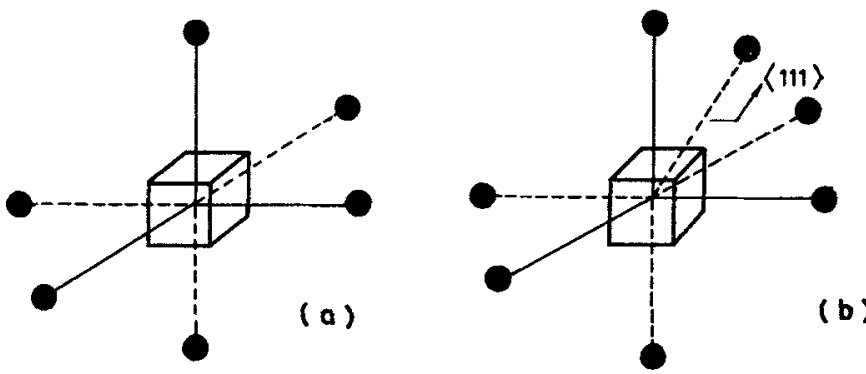

Figure 4. Minimum energy configurations of six and seven helium atoms in a single vacancy in $\mathrm{Cu}$. Helium atoms are described by filled circles and the vacancy by the empty cube.
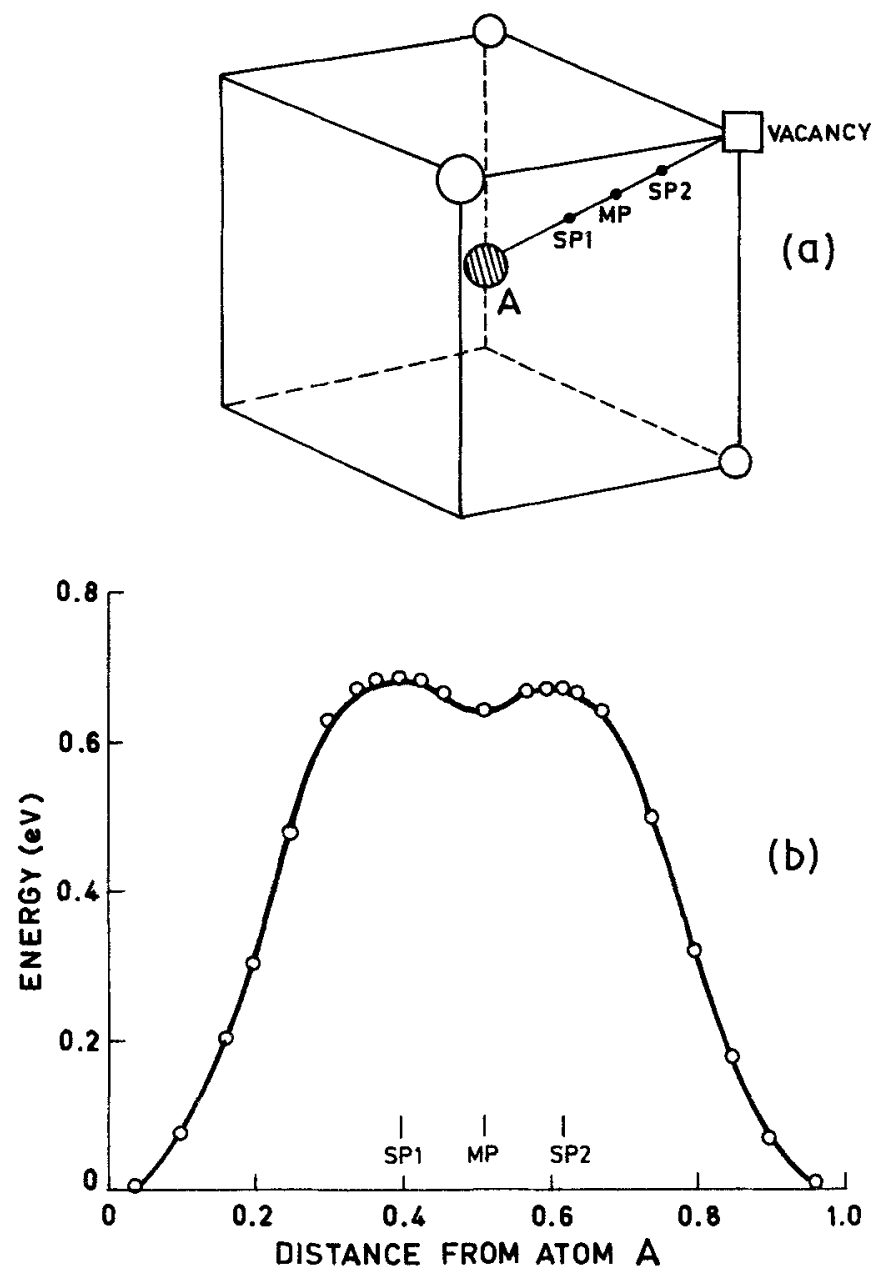

Figure 5. (a) Vacancy migration in bec iron. The vacancy ( $D$ ) migrates to the site $A$ (the body centre position) via the two saddle points SP1 and SP2. MP is the mid-point of the path. (b) Crystal configuration energy change for the vacancy migration path shown above. 
Computer simulation has also been employed in the study of dislocations and their interaction with point defects. For example the splitting into partials of an edge dislocation in an foc lattice has been seen by simulation. Quantities like the stacking fault width and the core structure have been evaluated (Perrin et al 1972). A most recent review covering the literature of dislocation modelling in various systems can be found in Puls (1980).

The study of dislocation-point defect interaction is very important to understand many metallurgical processes like strain aging etc. Computer simulation has been effectively utilized to obtain information regarding the configuration and energetics of this interaction. The interaction of a carbon atom with an edge dislocation in fcc nickel has been studied by Purniah et al (1985). The salient features of these results are the correct prediction that the small-sized interstitial prefers to sit above the slip plane. Further, the dipole tensor which was originally spherical in the absence of the dislocation becomes monoclinic.

\subsection{Displacement damage simulation}

Most of our knowledge about atomic displacements and cascade damage has come from dynamic computer simulation. A projectile with a given energy and appropriate direction with reference to the crystallographic orientations is allowed to move at time $t=0$. The subsequent interactions with the lattice atoms are then observed according to the predetermined force laws. Different structures emerge depending on the kinetic energy of the projectile. At energies less than the displacement energy $E_{\mathrm{d}}$ (typically $10-50 \mathrm{eV}$ in most metals), the energy loss is mainly by elastic collisions. This energy loss is channelled normally along crystallographic directions called focussons (Silsbee 1957). At energies around $E_{d}$ and above, a Frenkel pair is formed. One of the important features of this process is the replacement collision sequence where atoms in a chain exchange positions. The length of such sequences is generally 3 to 10 atomic distances but rather long replacement collision chains (greater than 50 atomic distances) can also exist in certain close packed directions (Lucasson 1975). The minimum displacement energy $E_{d}$ depends on the relative direction of the projectile with respect to the crystallographic planes and is often obtained as the displacement energy surface as shown in figure 6a. At higher projectile energies the probability of forming more than one Frenkel defect increases (figure 6b). At still higher energies new features emerge. The initial PKA is able to impart enough kinetic energy to atoms to produce secondary collision cascades which normally branch off at right angles to the primary cascades thus resulting in multiple Frenkel pairs. These subcascades can sometimes overlap reducing somewhat the chances of survival of Frenkel pairs. In all these simulation runs the relaxation of atoms, spontaneous reorganization or recombination of unstable configurations is built into the simulation event. A graphical picture of the process at $5 \mathrm{keV}$ obtained by Beeler (1966) is given in figure 7.

\section{Conclusions}

In this paper we have discussed the computer simulation technique and its application to the study of defects in materials. In particular, we have considered the case of point defects, impurities, dislocations and dislocation-point defect interactions. Several other 


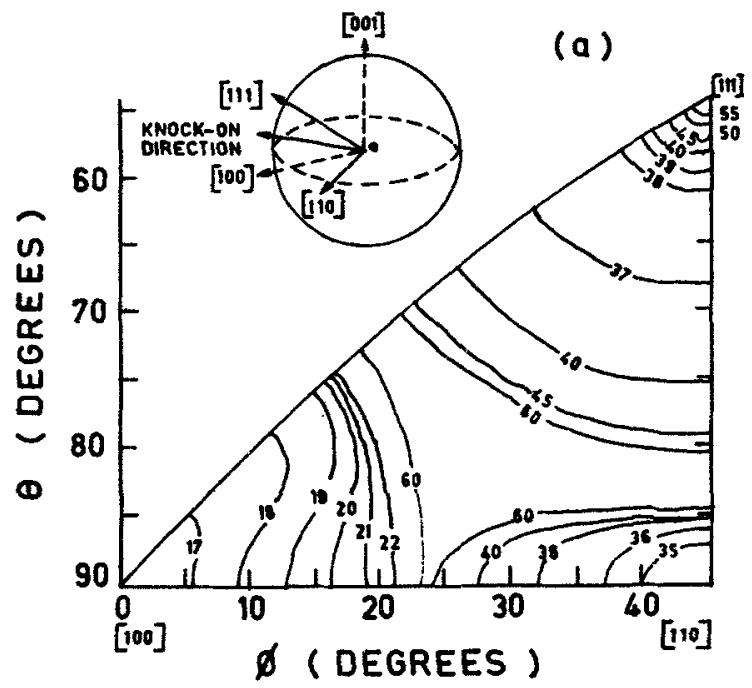

(b)

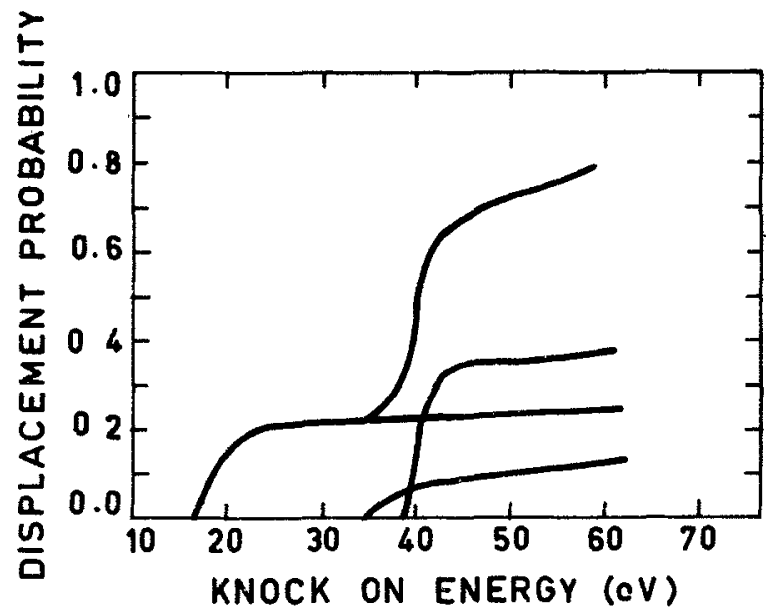

Figure 6. (a) Contours of constant displacement energy threshold (in $\mathrm{eV}$ ) in boc Fe. The polar and azimuthal angles define the PKA emission direction (Erginsoy et al 1964). (b) Integrated displacement probability $P_{d}(t)$ for a PKA of energy $E$ and random direction in boc iron. Contributions to the three low-index directions are shown separately.

areas of study have been omitted due to the limitations of space. Notable among these are studies regarding the behaviour of grain boundaries, slip and other deformation related processes. While the results depend very much on the exact interatomic potential chosen, the wide applicability of this technique makes it a very useful tool of research. 


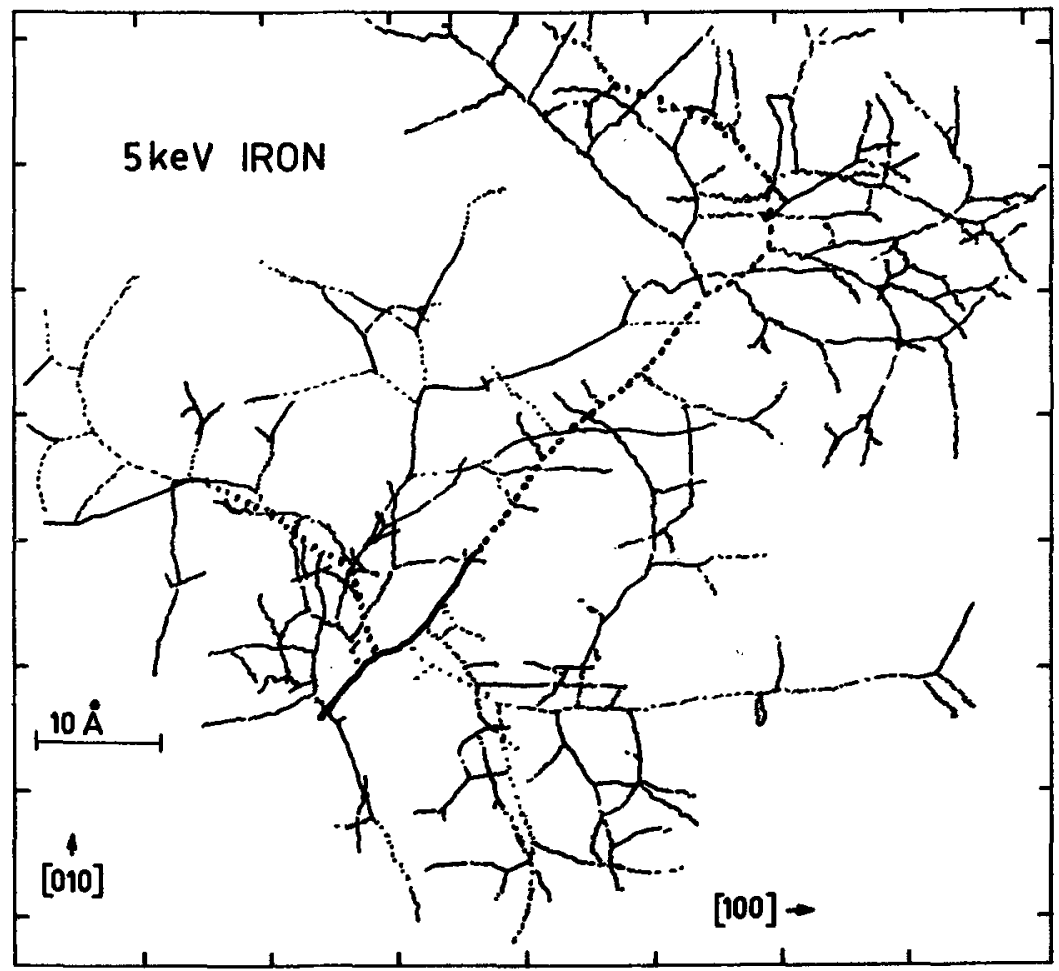

Figare 7. Trajectory map for a $5 \mathrm{keV}$ collision crack in bce iron. The short thick line represents the PKA trajectory. The 3 main secondary knock on trajectories are represented by heavy dotted lines. For higher order KA the trajectory lines are alternately solid and dashed lines.

\section{References}

Baskes M I and Melius C F 1979 Phys. Rev. B20 3197

Beeler J R Jr 1966 Phys. Rev. 150470

Beeler J R Jr 1983a Radiation effects computer experiments (Amsterdam: North-Holland)

Beeler J R Jr 1983b Radiation effects computer experiments (Amsterdam: North-Holland) p. 318

Beeler J R Jr 1983c Radiation effects computer experiments (Amsterdam: North-Holland) p. 428

Beeler J R Jr and Johnson R A 1967 Phys. Rev. 156677

Cotterill R M J and Doyama M 1967 in Lattice defects and their interactions (ed.) R R Hasiguti (New York:

Gordon and Breach) p. 20

Erginsoy C, Vineyard G H and Englert A 1964 Phys. Rev. A133 595

Gibson J B, Goland A N, Milgrom M and Vineyard G H 1960 Phys. Rev. 1201229

Hoagland R G, Hirth J P and Gehlen P C 1976 Philos. Mag. 34413

Johnson R A 1973 J. Phys. F3 295

Lucasson P 1975 in Fundamental aspects of radiation in metals (eds) M T Robinson and F W Young (US Department of Commerce, National Technical Information Service, Springfield, USA, Report Conf. 751006) Vol. 1, p. 42

Perrin R C, Englert A and Bullough R 1972 in Interatomic potentials and simulation of lattice defects (eds.) P C Gehlen, J R Beeler Jr. and R I Jaffee (New York: Plenum Press) p. 509

Puls M P 1980 in Proc. Int. Conf. on Dislocation Modelling of Physical Systems, (ed) C S Hartley (UK: Pergamon Press) Special Acta Met./Scripta Met. Publication 
Purniah B, Srinivasan S and Krishan K 1980 in Proc. Nucl. Phys. and Sol. State Phys. Symposium, Delhi (Department of Atomic Energy) Vol. C23, p. 742

Purniah B, Srinivasan S and Krishan K 1982 in Point defects and defect interactions in metals Proc. Int. Conf., (eds) J Takamura, M Doyama and M Kiritani (Tokyo: Univ. of Tokyo Press) p. 322

Purniah B and Srinivasan S 1986 Bull. Mater. Sci. 8247

Sahu H K, Srinivasan S and Krishnan K 1980 Pramana (J. Phys.) 15189

Silsbee R H 1957 J. Appl. Phys. 281246

Wilson W D, Baskes M I and Bisson C L 1976 Phys. Rev. B13 2470 